\title{
Endoscopic ultrasound-guided intratumoural therapy for pancreatic cancer
}

\author{
Brian M Yan MD FRCPC ${ }^{1}$, Jacques Van Dam MD PhD ${ }^{2}$
}

BM Yan, J Van Dam. Endoscopic ultrasound-guided intratumoural therapy for pancreatic cancer. Can J Gastroenterol 2008;22 (4):405-410.

Pancreatic cancer is the second most frequent gastrointestinal malignancy and carries a dismal prognosis. The current standard of care includes resection, if possible, as well as systemic chemoradiation therapy. Endoscopic ultrasound (EUS) is an established technique for the diagnosis and staging of pancreatic adenocarcinoma. Interventional EUS via fine needle injection (FNI) for the treatment of pancreatic cancer is a rapidly expanding field. The present article reviews the up-to-date developments in EUS FNI for intratumoural pancreatic cancer therapy, including antitumoural agents, immunotherapy, ablative techniques and new delivery systems. The therapeutic modalities discussed are currently under development and will hopefully reach clinical practice if benefit is demonstrated through clinical trials. EUS FNI may be an exciting new technique for the delivery of desperately needed novel therapies for pancreatic cancer.

Key Words: Brachytherapy; Cytoimplant; Dendritic cells; Endoscopic ultrasound; Fiducials; Fine needle injection; ONYX-015; Pancreatic cancer; Photodynamic therapy; Radiofrequency ablation; TNFerade

\section{Une thérapie intratumorale du cancer du pancréas, orientée par échoendoscopie}

Le cancer du pancréas est le deuxième cancer gastro-intestinal le plus fréquent, et son pronostic est lugubre. La norme actuelle de soins inclut une résection, dans la mesure du possible, ainsi qu'une chimioradiothérapie systémique. L'échoendoscopie est une technique établie pour poser le diagnostic et établir la phase de l'adénocarcinome du pancréas. L'échoendoscopie interventionniste par injection à l'aiguille est en rapide expansion dans le traitement du cancer du pancréas. Le présent article analyse les développements à jour de l'échoendoscopie à l'aiguille dans la thérapie intratumorale du cancer du pancréas, y compris les agents antitumoraux, l'immunothérapie, les techniques ablatives et les nouveaux systèmes de libération de médicaments. Les modalités thérapeutiques abordées sont en voie de développement, et on espère qu'elles seront utilisées en pratique clinique si des essais cliniques en démontrent l'efficacité. L'échoendoscopie à l'aiguille pourrait être une nouvelle technique captivante pour l'administration de nouvelles thérapies dont on a désespérément besoin pour le cancer du pancréas.
Dancreatic adenocarcinoma is the second most frequent gastrointestinal malignancy and the fourth leading cause of cancer mortality in the United States $(1,2)$. Overall survival is dismal, with a one- and five-year survival of $20 \%$ and less than $4 \%$, respectively (1). Resection offers the best chance for patients to be cured. For the minority of patients who have resectable disease (15\%), five-year survival is improved to $20 \%$ to $25 \%$ (3).

All patients with pancreatic cancer may benefit from systemic therapy. Adjuvant chemoradiation therapy with 5-fluorouracil improves survival compared with surgery alone $(4,5)$. Furthermore, neoadjuvant chemoradiation may reduce locoregional recurrence (6). Gemcitabine offers a modest improvement over 5-fluorouracil in those with unresectable disease and, in the adjuvant setting, postcurative resection $(7,8)$. A recent Cochrane analysis (9) suggested that chemotherapy improves survival and quality of life in patients with advanced pancreatic cancer. However, current evidence does not suggest superiority of multiagent chemotherapy or combination chemoradiation therapy over single-agent chemotherapy alone (9). Most recent data suggest a benefit from the use of a protein tyrosine kinase inhibitor (eg, erlotinib) combined with gemcitabine (10). Despite a marginal benefit, the overall response of pancreatic cancer to current systemic therapy unfortunately continues to be poor, and new therapies are desperately needed $(11,12)$.

\section{ENDOSCOPIC ULTRASOUND AND PANCREATIC CANCER}

Endoscopic ultrasound (EUS) is a highly sensitive and accurate modality for the diagnosis and staging of pancreatic cancer (1318). The position of the echoendoscope transducer within the stomach or duodenum allows for unparalleled access to the pancreas. With a curvilinear echoendoscope, a needle can be passed through the working channel under ultrasound guidance directly into a tumour or lymph node to obtain tissue for diagnosis. Fine needle aspiration (FNA) increases the diagnostic accuracy of EUS to 95\% (14). Fine needle injection (FNI) has naturally emerged from this technique, enabling therapeutic modalities under real time EUS guidance (Figure 1). Thus, the field of 'interventional' EUS is rapidly expanding from a diagnostic modality to the therapeutic management of pancreatic

\footnotetext{
${ }^{1}$ Division of Gastroenterology, University of Calgary, Calgary, Alberta; ${ }^{2}$ Division of Gastroenterology, Department of Medicine, Stanford University Medical Center, Stanford, California, USA

Correspondence: Dr Brian Yan, Division of Gastroenterology, University of Calgary, Room 6D16 TRW Building, 3280 Hospital Drive

Northwest, Calgary, Alberta T2N 4N1. Telephone 403-592-5035, fax 403-592-5090, e-mail bmyan@ucalgary.ca

Received for publication August 31, 2007. Accepted November 26, 2007
} 


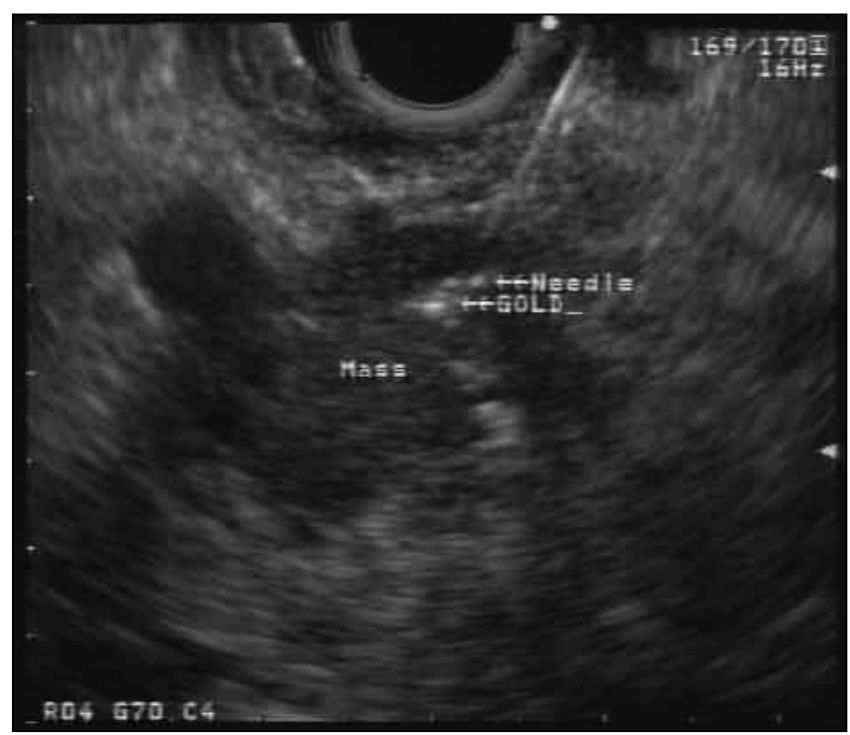

Figure 1) Linear endoscopic ultrasound with a 19-gange needle placed in a hypoechoic pancreatic mass for the insertion of gold fiducials. This patient had unresectable adenocarcinoma of the pancreas and underwent image-guided radiation therapy in combination with gemcitabine

cancer (19-21). EUS FNI provides a safe and minimally invasive method for direct intratumoural delivery of therapeutic agents.

\section{EUS FNI ANTITUMOUR THERAPY FOR PANCREATIC ADENOCARCINOMA}

Many novel therapeutic agents and techniques delivered via EUS FNI have been used in clinical trials for the treatment of advanced pancreatic cancer. Injectable agents include activated lymphocyte cultures, viral vectors and oncolytic viruses. All agents were solutions that were directly injected into pancreatic tumours through a standard EUS FNA needle. Intratumoural coagulative techniques have also been attempted via access through the EUS needle. Table 1 summarizes the therapies studied.

\section{EUS injectable anti-tumour agents}

Cytoimplant: Chang et al (22) completed a phase I trial in which eight patients with advanced pancreatic cancer were given intratumoural injections of activated allogenic mixed lymphocyte culture (cytoimplant). The cytoimplant was generated from a co-culture of healthy donor and patient peripheral blood mononuclear cells. Doses of $3 \times 10^{9}, 6 \times 10^{9}$ or $9 \times 10^{9}$ cells were given in a single injection session, with no procedurerelated complications. The most common side effect was fever. Grade 3 to 4 toxicities occurred in seven patients, including elevated liver enzymes and bilirubin, and dehydration secondary to nausea and vomiting. Dose-limiting toxicity, however, was not reached because all grade 3 to 4 toxicities were reversible. No patient had treatment-related pancreatitis. Tumour response was followed by computed tomography or EUS: two patients had a partial response (ie, more than a 50\% decrease in cross-sectional area), one had a minimal response, three had no change and two had a progression of their disease. Median survival was 13.2 months (range 4.2 to more than 36 months). Interim results of a randomized trial comparing the cytoimplant with gemcitabine suggested a worse outcome in patients who had the cytoimplant. The trial was suspended and final results have not been published.

TNFerade: TNFerade (GenVec Inc, USA) is a replicationdeficient adenovirus vector carrying the human tumour necrosis factor-alpha gene regulated by a radiation-inducible promoter (Egr-1). Intratumoural TNFerade with radiation has been shown to be safe in a phase I clinical trial of 30 patients with solid tumours (23). A phase I/II multicentre pancreatic cancer trial was conducted, using TNFerade in combination with continuous intravenous 5 -fluorouracil $\left(200 \mathrm{mg} / \mathrm{m}^{2} /\right.$ day $\times$ five days/week) and radiation (50.4 Gy). TNFerade was delivered intratumourally by EUS, computed tomography or percutaneous ultrasound (PTA) guidance. The complete results are yet to be published; however, preliminary data are available for 50 patients, of which 27 and 23 patients had EUS- and PTA-guided treatment, respectively (24). Partial response occurred at three months in $13 \%$ of patients who had EUS-guided treatment versus $10 \%$ of patients who had PTAguided treatment. Stabilization occurred in $73 \%$ of EUS patients versus $75 \%$ of PTA patients, and overall survival without progression occurred in $53 \%$ of EUS patients versus $47 \%$ of PTA patients. Higher maximal tolerated doses of up to $4 \times 10^{11}$ pu were associated with greater locoregional control, a greater proportion of patients with stable or decreasing carbohydrate antigen 19-9, a greater percentage of patients having resections and longer progression-free and median survival. Dose-limiting toxicity was seen in one PTA patient (hypotension) and three EUS patients (pancreatitis in two patients, and one biliary obstruction). Other grade 1 to 2 toxicities included fever $(36 \%)$, rigors $(25 \%)$, fatigue (18\%), nausea and vomiting $(16 \%)$, and anorexia (12\%). TNFerade delivery did not interfere with surgical resection. A randomized phase II/III study is underway and an interim analysis of the first 50 patients (target accrual of 330 patients) was presented at the American Society of Clinical Oncology 43rd Annual Meeting in 2007 (25). The standard of care (SOC) included continuous infusion of 5-fluorouracil and radiation followed by maintenance gemcitabine or gemcitabine/erlotinib. Patients with locally advanced unresectable pancreatic adenocarcinoma were randomly assigned to receive either TNFerade plus SOC or SOC alone. Median survival was 19.3 months for TNFerade/SOC, compared with 11.1 months for SOC alone. There was a trend toward improved one-year and overall survival; however, this trend was not statistically significant. The tumour response at three months was $73 \%$ stable disease and $27 \%$ progressive disease in patients treated with TNFerade plus SOC. Patients treated with SOC alone had a $6 \%$ partial response, $75 \%$ stable disease and $19 \%$ progressive disease. The difference in tumour stabilization was not statistically significant. A second interim analysis is anticipated.

ONYX-015: ONYX-015 (Onyx Pharmaceuticals, USA) is an oncolytic attenuated adenovirus that preferentially replicates in malignant cells, leading to cell death (26). Hecht et al (27) completed a phase I/II trial of EUS FNI-guided intratumoural delivery of ONYX-015 combined with gemcitabine in 21 patients with advanced pancreatic carcinoma. The FNI protocol consisted of $1 \mathrm{~mL}$ of virus per injection, with up to 10 injections per session depending on tumour size, for a total of eight sessions over eight weeks. The total particle dose per session was $2 \times 10^{10}$ pu to a maximum of $2 \times 10^{11} \mathrm{pu}$, depending on toxicity experienced with lower doses. Gemcitabine infusion was given on the same day as the last four injections. 
TABLE 1

Endoscopic ultrasound (EUS)-guided fine needle injection (FNI) antitumoural therapies for pancreatic cancer

\begin{tabular}{|c|c|c|c|c|}
\hline Reference, year & Therapy & $\mathbf{n}$ & Tumour response (\%) & Adverse events \\
\hline \multirow[t]{3}{*}{ Chang et al (22), 2000} & \multirow[t]{3}{*}{ Cytoimplant } & \multirow[t]{3}{*}{8} & Partial (25) & Fever \\
\hline & & & Minimal (12) & Elevated liver enzymes/bilirubin \\
\hline & & & Progression (25) & \\
\hline \multirow{3}{*}{$\begin{array}{l}\text { Farrell et al (24) (Abst), } \\
2006\end{array}$} & \multirow[t]{3}{*}{ TNFerade } & \multirow[t]{3}{*}{51} & Partial (13) & Fever \\
\hline & & & \multirow[t]{2}{*}{ Stable (73) } & Rigors \\
\hline & & & & Anorexia \\
\hline \multirow[t]{2}{*}{ Posner et al* (25) (Abst), 2007} & \multirow[t]{2}{*}{ TNFerade } & \multirow[t]{2}{*}{51} & Stable $(73)$ & \multirow[t]{2}{*}{ Neutropenia } \\
\hline & & & Progressive (27) & \\
\hline \multirow[t]{2}{*}{ Hecht et al (27), 2003} & \multirow[t]{2}{*}{ ONYX-015 (Onyx Pharmaceuticals, USA) } & \multirow[t]{2}{*}{21} & Partial (10) & Duodenal perforation \\
\hline & & & Minor (10) & Sepsis \\
\hline \multirow{3}{*}{ Goldberg et al (28), 1999} & \multirow{3}{*}{ Radiofrequency ablation } & \multirow{3}{*}{8 (porcine) } & \multirow{3}{*}{$N / A$} & Hyperamylasemia \\
\hline & & & & Pseudocyst \\
\hline & & & & Serosal burns \\
\hline Chan et al (30), 2006 & Photodynamic therapy & 3 (porcine) & $\mathrm{N} / \mathrm{A}$ & None stated \\
\hline \multirow[t]{3}{*}{ Sun et al (31), 2006} & \multirow[t]{3}{*}{ Brachytherapy (iodine-125) } & \multirow[t]{3}{*}{15} & Partial (27) & Pancreatitis \\
\hline & & & Minimal (20) & Pseudocyst \\
\hline & & & Stable $(30)$ & Hematological \\
\hline \multirow[t]{3}{*}{ Jin et al (32) (Abst), 2007} & \multirow[t]{3}{*}{ Brachytherapy (iodine-125) } & 28 & Partial (11) & \multirow[t]{3}{*}{ Fever } \\
\hline & & (25 pancreatic) & Stable (43) & \\
\hline & & & Progressive (36) & \\
\hline
\end{tabular}

Summary of studies for EUS-guided antitumoural therapies for pancreatic cancer. ${ }^{*}$ This study is the only randomized controlled trial currently ongoing. Patients are randomly assigned to receive either TNFerade (GenVec Inc, USA) with standard of care (SOC) or SOC alone. For the first 51 patients enrolled (of a target total of 330), no statistical significance was found between the TNFerade plus SOC group versus the SOC alone group in overall, median and one-year survival, or in tumour response. Only one adverse event (ie, neutropenia) occurred in more than $15 \%$ of patients and was more frequent in patients who received TNFerade plus SOC compared with SOC alone. N/A Not applicable

Two patients developed sepsis and two duodenal perforations occurred. All four complications were attributed to the EUS FNI technique, and were corrected with protocol adjustments, including a strictly transgastric approach, alteration of needle withdrawal technique between injections and prophylactic antibiotics. No convincing efficacy for ONYX-015 was found: two with partial regression; two with minor progression; six with stable disease; and 11 with progressive disease or treatmentrelated toxicity. The median survival was 7.5 months. Nonetheless, the investigators demonstrated safety and tolerability of repetitive EUS-guided FNI.

EUS-guided coagulative therapies

Radiofrequency ablation: Radiofrequency ablation (RFA) provides predictable coagulative necrosis to a defined area, with relatively low morbidity compared with surgical intervention. It has primarily been administered percutaneously or intraoperatively to patients with hepatocellular carcinoma and metastases to the liver, brain or bone. Goldberg et al (28) studied the safety of EUS-guided RFA in a pig model. A needle electrode was passed transgastrically under EUS guidance to the tail of the pancreas in 13 Yorkshire pigs. Energy was supplied by a $500 \mathrm{kHz}$ monopolar RF generator, through an insulated metal needle with only its distal $1 \mathrm{~cm}$ to $1.5 \mathrm{~cm}$ tip left uninsulated. Radiofrequency was applied for $6 \mathrm{~min}$ with a goal tip temperature of $90^{\circ} \mathrm{C}$. The first five pigs were euthanized after ablation, and pathology showed a well-demarcated acute coagulation zone of $8 \mathrm{~mm}$ to $10 \mathrm{~mm}$ in all specimens. Two of these pigs had gastric burns, but no frank perforation. The other eight pigs were followed for 14 days, showing progression from subacute coagulation, to walled off coagulation, to fibrosis. On day 2, one pig had mild hyperamylasemia and had a subsequent pseudocyst formation at day 14; another had an intestinal serosal burn at day 14. EUS-guided RFA may be a potential modality for the treatment of small neuroendocrine tumours, poorly accessible liver lesions or unresectable pancreatic adenocarcinoma.

Photodynamic therapy: Photodynamic therapy (PDT) involves the administration of a photosensitive agent that is activated by light in the presence of oxygen. Photochemical 
tissue necrosis occurs rather than an ionizing thermal burn (ie, RFA), resulting in tumour necrosis but minimal damage to connective tissue. Brown et al (29) conducted a phase I study in 16 patients with inoperable pancreatic adenocarcinoma. PDT was delivered percutaneously, and results showed that all had tumour necrosis on repeat imaging. Two patients with gastroduodenal artery involvement had significant gastrointestinal bleeding. Three patients developed duodenal obstruction. The median survival was 9.5 months, with 44\% alive after one year. Chan et al (30) studied EUS-guided PDT in a pig model. Three pigs were pretreated with porfimer sodium and a 19-gauge fine needle was passed into the liver, kidney, spleen and pancreas under EUS guidance. Light was supplied through a small diameter quartz optical fibre with a $1.0 \mathrm{~cm}$ cylindrical light diffuser that was passed through the needle into the tissue. Fifty joules of $630 \mathrm{~nm}$ light over $125 \mathrm{sec}$ was delivered per treatment. In total, there were 26 treatment locations, of which nine were in the pancreas. Localized tissue necrosis was seen in all organs treated. The area of necrosis in the pancreas was $3.6 \mathrm{~mm}^{2}$, of which $100 \%$ had complete necrosis, $44 \%$ had a hemorrhage present, and $67 \%$ had granulation tissue. There were no significant procedure-related complications or pancreatitis. EUS-guided low-dose PDT may therefore be safe and feasible for benign or malignant lesions of the pancreas, liver, kidney or spleen.

Brachytherapy: Brachytherapy has been successfully used in a number of solid organ tumours. Radioactive seeds, including iodine-125 $\left({ }^{125} \mathrm{I}\right)$ and palladium-103 $\left({ }^{103} \mathrm{Pd}\right)$, are placed directly into tumours or lymph nodes. A pilot study by Sun et al (31) in 15 patients with unresectable pancreatic cancer demonstrated the safety and feasibilty of EUS-guided brachytherapy. A mean of 22 seeds $\left({ }^{125} \mathrm{I}\right)$ were inserted under EUS guidance for a mean total activity of $20 \mathrm{mCi}$. Twentyseven per cent had a partial response, $20 \%$ had a minimal response, and $33 \%$ had stable disease. Clinical benefit was seen in $30 \%$ of patients, primarily from reduction of pain. Three patients experienced pancreatitis and pseudocyst formation, and three patients had grade 3 hematological toxicity. A similar study by Jin et al (32) has recently been presented in abstract form. Twenty-eight patients with abdominal malignancies were treated with ${ }^{125} \mathrm{I}$ brachytherapy in combination with chemotherapy. Twenty-five patients had advanced pancreatic cancer, while the other three patients had metastases (cholangiocarcinoma, gastric cardia adenocarcinoma and an unknown secondary adenocarcinoma). After a median follow-up time of 5.4 months, only three cases (10.7\%) had a partial response, while 12 had stable disease, 10 had progressive disease and three were lost to follow-up. There were no serious complications related to the procedures; however, $54 \%$ of the patients developed mild fever in the first $24 \mathrm{~h}$. Particle-based brachytherapy using phosphorus$32\left({ }^{32} \mathrm{P}\right)$ in two patients has also been reported in abstract form (33). This may improve the distribution of radioactive material through the tumour, solve the mechanical difficulties of inserting solid seeds and possibly improve efficacy.

\section{FUTURE EUS-GUIDED FNI ANTITUMOURAL THERAPIES}

A multitude of agents are in development that potentially can be delivered by EUS FNI. Broad targets include inhibitors of oncogenes with antisense oligonucleotides or competitive RNA, replacement of tumour suppressor genes, inhibition of cell signalling pathways, gene-directed prodrug activation, replication competent oncolytic viruses, angiogenesis inhibitors and apoptosis promoters (34). While most only show moderate anti-tumoural activity in early in vitro and in vivo trials, combinations of treatments or alterations of dose and route of delivery may improve efficacy.

Innovative drug delivery systems may potentially improve the efficacy of locally injected chemotherapeutic or antitumoural agents. The ReGel drug delivery system (Macromed Inc, USA) is a thermosensitive biodegradable polymer. At body temperature, the injected water-soluble polymer is transformed to a water-insoluble hydrogel that continuously releases the drug to the target organ. Matthes et al (35) have demonstrated localized, sustained high concentrations of paclitaxel in the pancreata of pigs when injected with the OncoGel (ReGel combined with paclitaxel) delivery system (Protherics, United Kingdom). Solid polymers embedded with 5-fluorouracil have been inserted into canine pancreata (36). Local tissue necrosis was demonstrated without significant complications to the animals. Newly developed nanotubes have been recently reported in abstract form for the delivery of gadolinium to enhance the magnetic resonance imaging signal in porcine pancreata (37). Conceivably, this technology can also be used to deliver antitumour therapy. All of these new systems may prove to be an effective means of targeted drug delivery, thereby increasing local drug concentrations and efficacy.

EUS-guided FNI may have a supportive role in targeted radiation therapy. Image-guided radiotherapy (IGRT) is a method of delivering highly conformal radiation treatments, thereby causing minimal toxicity to surrounding tissue. Initially developed more than 50 years ago for tumours of the brain and central nervous system, IGRT has more recently been applied to non-central nervous system tumours with fiducials as reference points for radiation beam tracking and delivery. Koong et al $(38,39)$ have demonstrated excellent local control in phase I/II studies of IGRT in patients with locally advanced pancreatic cancer, albeit with no change in overall survival. Gold fiducials were placed laparoscopically or with computed tomography guidance. EUS-guided gold fiducial placement in abdominal and mediastinal malignancies has been shown to be safe and feasible $(40,41)$. Presently, there are no data comparing methods of fiducial placement; however, EUS offers the advantages of a relatively low risk procedure and technical ease of insertion. At our centre (Stanford University Medical Center, Stanford, California, USA), all patients undergoing IGRT for advanced pancreatic cancer have fiducials placed by EUS FNI.

Immunotherapy for pancreatic cancer treatment is a novel concept based on progressive knowledge regarding the role of the immune system in cancer surveillance and control. Dendritic cells (DCs) are extremely potent tumour antigenpresenting cells and are known to have numerous critical immunoregulatory functions (42). Tumours may escape immune surveillance by DC inactivation (43) or due to a lack of DCs (44). Prior in vitro studies have shown that DCs exposed to tumour cell lysates from human pancreatic carcinoma cell lines induce a strong $\mathrm{T}$ cell response against the cancer cells (45). Translational research for cancer treatment in this field, however, is complicated by the many factors involved in DC generation. Conditions of DC generation, maturation state, activating factors, timing of injection and route of injection all may significantly affect tumoural 
response. Nonogaki et al (46) presented the first abstract for dendritic cell therapy in human pancreatic cancer at Digestive Disease Week 2007. Five patients with locally advanced pancreatic cancer were treated with gemcitabine and biweekly EUS-guided injection of immature DCs. One patient had a partial tumour response, allowing curative resection, and two other patients had stable disease for over six months. Grade 2 leukopenia occurred in one patient. Our centre is currently conducting a phase I/II trial of EUS-guided FNI intratumoural dendritic cell immunotherapy in combination with chemoradiation therapy. Dendritic cell therapy may have a significant role in the future therapy of pancreatic cancer. An even more intriguing concept of this therapy is the possibility of long-term immunity (ie, vaccination effect) against adenocarcinoma.

\section{SUMMARY}

Effective treatment for pancreatic cancer is urgently needed. EUS has played a key role in the diagnosis and staging of disease. With advancing technology, EUS with FNI can now provide therapeutic modalities by direct intratumour delivery of antitumour agents. Studies to date have demonstrated the safety and feasibility of various EUS-guided FNI therapies; however, no large efficacy trials have been completed. Early studies with cytoimplant and ONYX-015 unfortunately seem ineffective as single agents. The efficacy of TNFerade appears to be promising, and larger randomized trials are underway. Larger studies demonstrating the efficacy of EUS-guided coagulative therapies are needed. Immunotherapy with DCs shows promise, and may improve patient outcomes in the future. With its safety and ease of intratumoural delivery of therapeutic agents, EUS-guided FNI will play a key role in the future treatment of advanced pancreatic cancer.

ACKNOWLEDGEMENTS: Dr Brian M Yan is supported by a clinical research fellowship from the Canadian Institutes of Health Research.

\section{REFERENCES}

1. Greenlee RT, Hill-Harmon MB, Murray T, Thun M. Cancer statistics, 2001. CA Cancer J Clin 2001;51:15-36.

2. Reis L, Eisner M, Kosary C, et al. SEER Cancer Statistics Review, 1975-2002. Bethesda: National Cancer Institute, 2005.

3. Kuvshinoff BW, Bryer MP. Treatment of resectable and locally advanced pancreatic cancer. Cancer Control 2000;7:428-36.

4. Further evidence of effective adjuvant combined radiation and chemotherapy following curative resection of pancreatic cancer. Gastrointestinal Tumor Study Group. Cancer 1987;59:2006-10.

5. Klinkenbijl JH, Jeekel J, Sahmoud T, et al. Adjuvant radiotherapy and 5-fluorouracil after curative resection of cancer of the pancreas and periampullary region: Phase III trial of the EORTC gastrointestinal tract cancer cooperative group. Ann Surg 1999;230:776-82; discussion 782-4.

6. Hoffman JP, Lipsitz S, Pisansky T, Weese JL, Solin L, Benson AB III. Phase II trial of preoperative radiation therapy and chemotherapy for patients with localized, resectable adenocarcinoma of the pancreas: an Eastern Cooperative Oncology Group Study. J Clin Oncol 1998;16:317-23.

7. Burris HA III, Moore MJ, Andersen J, et al. Improvements in survival and clinical benefit with gemcitabine as first-line therapy for patients with advanced pancreas cancer: A randomized trial. J Clin Oncol 1997;15:2403-13.

8. Oettle H, Post S, Neuhaus P, et al. Adjuvant chemotherapy with gemcitabine versus observation in patients undergoing curativeintent resection of pancreatic cancer: A randomized controlled trial. JAMA 2007;297:267-77.
9. Yip D, Karapetis C, Strickland A, Steer CB, Goldstein D. Chemotherapy and radiotherapy for inoperable advanced pancreatic cancer. Cochrane Database Syst Rev 2006;3:CD002093.

10. Moore MJ, Goldstein D, Hamm J, et al. Erlotinib plus gemcitabine compared with gemcitabine alone in patients with advanced pancreatic cancer: A phase III trial of the National Cancer Institute of Canada Clinical Trials Group. J Clin Oncol 2007;25:1960-6.

11. Pino SM, Xiong HQ, McConkey D, Abbruzzese JL. Novel therapies for pancreatic adenocarcinoma. Curr Gastroenterol Rep 2004;6:119-25.

12. Rosemurgy AS, Serafini FM. New directions in systemic therapy of pancreatic cancer. Cancer Control 2000;7:437-51.

13. DeWitt J, Devereaux B, Chriswell M, et al. Comparison of endoscopic ultrasonography and multidetector computed tomography for detecting and staging pancreatic cancer. Ann Intern Med 2004;141:753-63.

14. Kochman ML. EUS in pancreatic cancer. Gastrointest Endosc 2002;56(4 Suppl):S6-S12.

15. Muller MF, Meyenberger C, Bertschinger P, Schaer R, Marincek B. Pancreatic tumors: Evaluation with endoscopic US, CT, and MR imaging. Radiology 1994;190:745-51.

16. Palazzo L, Roseau G, Gayet B, et al. Endoscopic ultrasonography in the diagnosis and staging of pancreatic adenocarcinoma. Results of a prospective study with comparison to ultrasonography and CT scan. Endoscopy 1993;25:143-50.

17. Brugge WR. The role of endoscopic ultrasound in the diagnosis and staging of pancreatic malignancy. US Gastroenterolgy Review 2006:37-41.

18. Gress FG, Hawes RH, Savides TJ, et al. Role of EUS in the preoperative staging of pancreatic cancer: A large single-center experience. Gastrointestinal endoscopy 1999;50:786-91.

19. Shami VM, Parmar KS, Waxman I. Clinical impact of endoscopic ultrasound and endoscopic ultrasound-guided fine-needle aspiration in the management of rectal carcinoma. Dis Colon Rectum 2004:47:59-65.

20. Fazel A, Draganov P. Interventional endoscopic ultrasound in pancreatic disease. Curr Gastroenterol Rep 2004;6:104-10.

21. Klapman JB, Chang KJ. Endoscopic ultrasound-guided fine-needle injection. Gastrointest Endosc Clin N Am 2005;15:169-177, x.

22. Chang KJ, Nguyen PT, Thompson JA, et al. Phase I clinical trial of allogeneic mixed lymphocyte culture (cytoimplant) delivered by endoscopic ultrasound-guided fine-needle injection in patients with advanced pancreatic carcinoma. Cancer 2000;88:1325-35.

23. Senzer N, Mani S, Rosemurgy A, et al. TNFerade biologic, an adenovector with a radiation-inducible promoter, carrying the human tumor necrosis factor alpha gene: A phase I study in patients with solid tumors. J Clin Oncol 2004;22:592-601.

24. Farrell JJ, Senzer N, Hecht JR, et al. Long-term data for endoscopic ultrasound (EUS) and percutaneous (PTA) guided intratumoral TNFerade gene delivery combined with chemoradiation in the treatment of locally advanced pancreatic cancer (LAPC). Gastrointestinal Endoscopy 2006;63:AB93. (Abst)

25. Posner M, Chang K, Rosemurgy A, et al. Multi-center, phase 2/3, randomized, controlled clinical trial using TNFerade gene delivery combined with chemoradiation in patients with locally advanced pancreatic cancer (LAPC). American Society of Clinical Oncology Annual Meeting. Chicago, June 2007. (Abst)

26. Ries SJ. Elucidation of the molecular mechanism underlying tumor-selective replication of the oncolytic adenovirus mutant ONYX-015. Future Oncol 2005;1:763-66.

27. Hecht JR, Bedford R, Abbruzzese JL, et al. A phase I/II trial of intratumoral endoscopic ultrasound injection of ONYX-015 with intravenous gemcitabine in unresectable pancreatic carcinoma. Clin Cancer Res 2003;9:555-61.

28. Goldberg SN, Mallery S, Gazelle GS, Brugge WR. EUS-guided radiofrequency ablation in the pancreas: Results in a porcine model. Gastrointest Endosc 1999;50:392-401.

29. Bown SG, Rogowska AZ, Whitelaw DE, et al. Photodynamic therapy for cancer of the pancreas. Gut 2002;50:549-57.

30. Chan HH, Nishioka NS, Mino M, et al. EUS-guided photodynamic therapy of the pancreas: A pilot study. Gastrointest Endosc 2004;59:95-9.

31. Sun S, Xu H, Xin J, et al. Endoscopic ultrasound-guided interstitial brachytherapy of unresectable pancreatic cancer: Results of a pilot trial. Endoscopy 2006;38:399-403. 
32. Jin Z, Li Z, Du Y, Jiang Y, Chen J. Endoscopic ultrasonographyguided interstitial implantation of iodine 125 seeds combined with chemotherapy in the treatment of unresectable abdominal carcinoma: A prospective study. Gastrointest Endosc 2007;65:AB105. (Abst)

33. Meenan J, Mesenas S, Douglas N, et al. EUS-delivered therapy for pancreatic cancer: Initial experience with targeted injection of $32 \mathrm{P}$ biosilicon. Gastrointest Endosc 2007;65:AB208. (Abst)

34. Bhattacharyya M, Lemoine NR. Gene therapy developments for pancreatic cancer. Best Pract Res Clin Gastroenterol 2006;20:285-98.

35. Matthes K, Mino-Kenudson M, Sahani DV, et al. EUS-guided injection of paclitaxel (OncoGel) provides therapeutic drug concentrations in the porcine pancreas (with video). Gastrointest Endosc 2007;65:448-53.

36. Sun S, Wang S, Ge N, et al. Endoscopic ultrasound-guided interstitial chemotherapy in the pancreas: Results in a canine model. Endoscopy 2007;39:530-34.

37. Vignesh S, Wilson L, Hartman K, et al. EUS FNI of gadoliniumloaded ultra-short carbon nanotubes (GDNT) into porcine pancreas boosts the MR T1 signal 25-fold. Gastrointest Endosc 2007;65:AB195. (Abst)

38. Koong AC, Le QT, Ho A, et al. Phase I study of stereotactic radiosurgery in patients with locally advanced pancreatic cancer. Int J Radiat Oncol Biol Phys 2004;58:1017-21.

39. Koong AC, Christofferson E, Le QT, et al. Phase II study to assess the efficacy of conventionally fractionated radiotherapy followed by a stereotactic radiosurgery boost in patients with locally advanced pancreatic cancer. Int J Radiat Oncol Biol Phys 2005;63:320-3.

40. Pishvaian AC, Collins B, Gagnon G, Ahlawat S, Haddad NG. EUS-guided fiducial placement for CyberKnife radiotherapy of mediastinal and abdominal malignancies. Gastrointest Endosc 2006;64:412-7.

41. Ellsmere JC, Mahadevan A, Kelleher T, Chuttani RAM, Pleskow DK. EUS-guided radiotherapy fiducials for upper gastrointestinal malignancies. Gastrointest Endosc 2007;65:AB208. (Abst)

42. Banchereau J, Paczesny S, Blanco P, et al. Dendritic cells: controllers of the immune system and a new promise for immunotherapy. Ann N Y Acad Sci 2003;987:180-7.

43. Vicari AP, Caux C, Trinchieri G. Tumour escape from immune surveillance through dendritic cell inactivation. Semin Cancer Biol 2002;12:33-42.

44. Dallal RM, Christakos P, Lee K, Egawa S, Son YI, Lotze MT. Paucity of dendritic cells in pancreatic cancer. Surgery 2002;131:135-8.

45. Schnurr M, Galambos P, Scholz C, et al. Tumor cell lysate-pulsed human dendritic cells induce a T-cell response against pancreatic carcinoma cells: An in vitro model for the assessment of tumor vaccines. Cancer Res 2001;61:6445-50.

46. Nonogaki K, Hirooka Y, Itoh A, et al. Combined treatment with immunotherapy and chemotherapy using endoscopic ultrasonography: A phase 1 trial as first line treatment in patients with locally advanced pancreatic carcinoma. Gastrointest Endosc 2007;65:AB207. (Abst) 


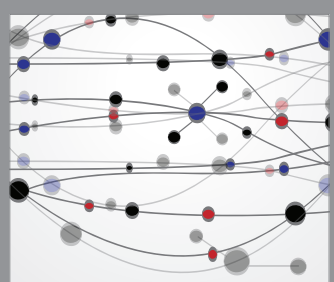

The Scientific World Journal
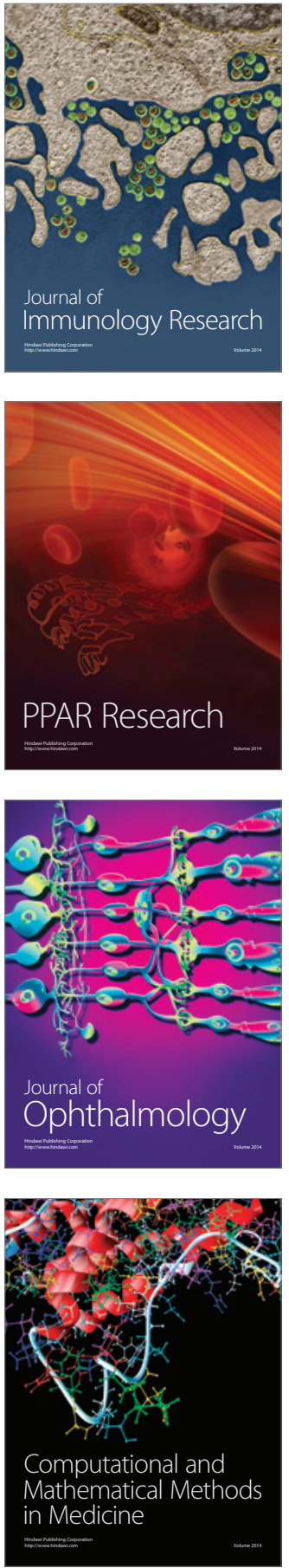

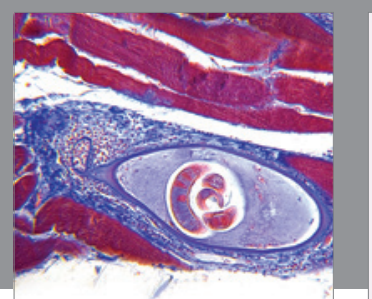

Gastroenterology Research and Practice

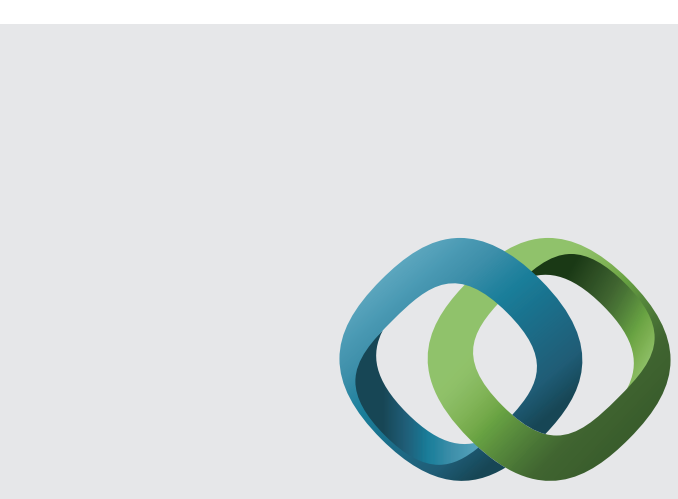

\section{Hindawi}

Submit your manuscripts at

http://www.hindawi.com
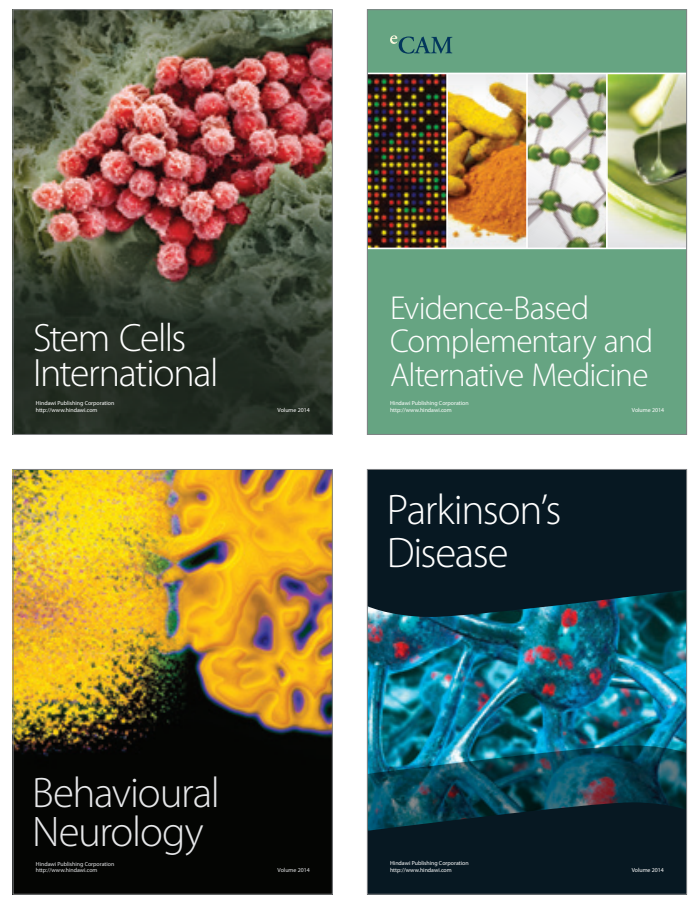
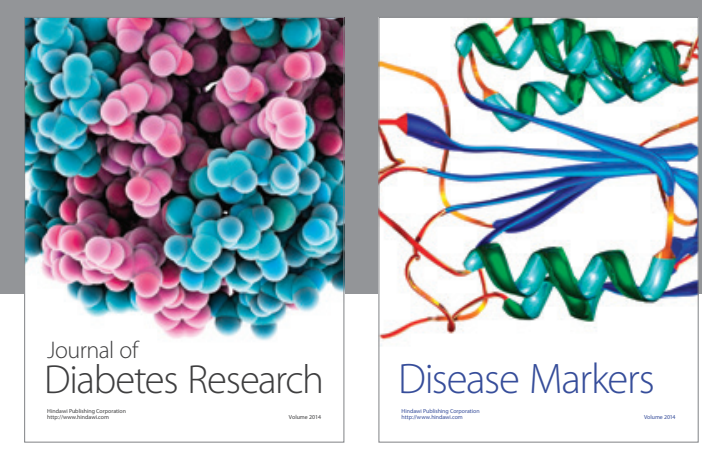

Disease Markers
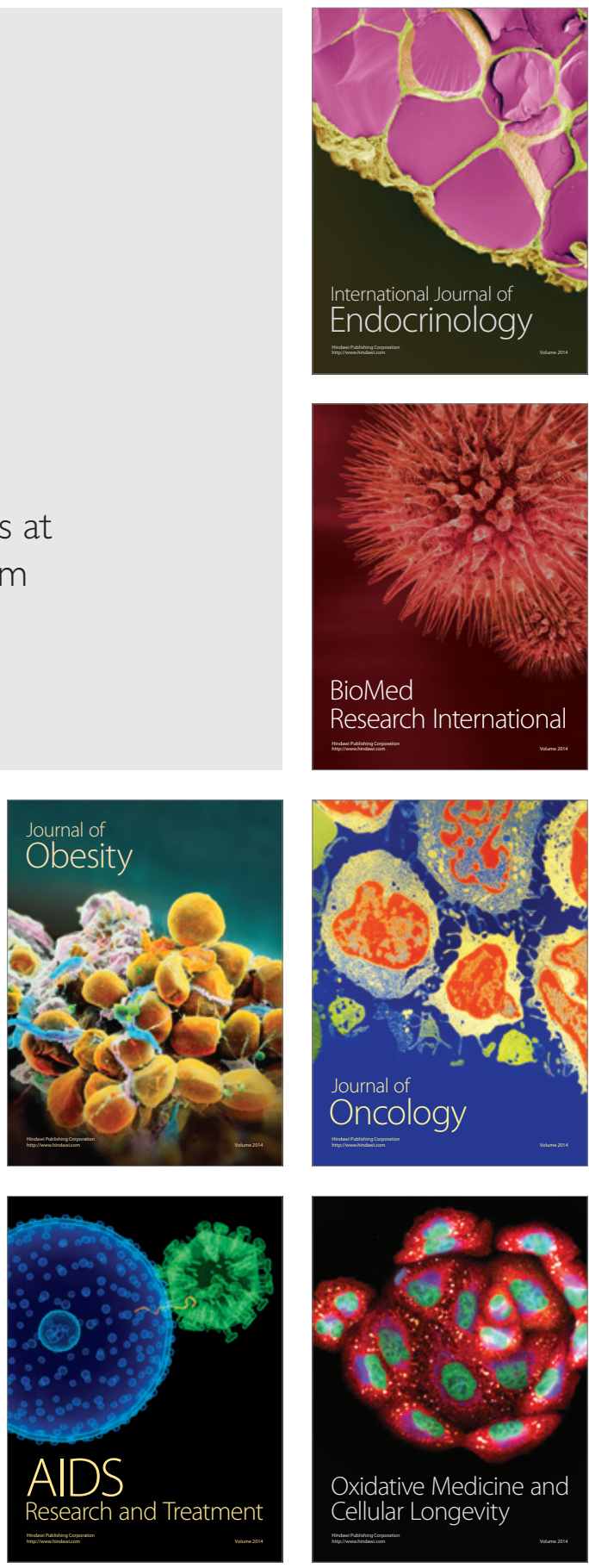